Semin Perinatol. 2013 February ; 37(1): 26-31. doi:10.1053/j.semperi.2012.11.001.

\title{
Ichthyosis in the Newborn
}

\author{
Brittany G. Craiglow, MD \\ aDepartment of Dermatology, Yale University, New Haven, CT
}

\begin{abstract}
The ichthyoses encompass a variety of genetic disorders marked by abnormal epidermal differentiation. The neonatal period is critical for patients with ichthyosis because of the risk for significant associated morbidity and mortality, with the majority of complications arising as a result of impaired barrier function. This article reviews presentations of ichthyosis in the neonate, outlines risks and complications, and provides strategies for management.
\end{abstract}

\section{Introduction}

The ichthyoses represent a large group of cutaneous disorders linked by the common finding of abnormal epidermal differentiation. The word ichthyosis comes from the Greek root ichthys, meaning fish, referring to the cutaneous scaling that is characteristic of these disorders, which is said to resemble the scales of a fish. Scaling can be localized or generalized and can be associated with a variety of additional cutaneous and/or systemic manifestations. Although these disorders are fairly rare, it is important for the perinatologist and neonatologist to have some familiarity with them, as the perinatal period and early infancy represent particularly critical stages for patients with ichthyosis.

In patients with ichthyosis, the barrier function of the skin is compromised and has a decreased ability to protect against bacterial, chemical, and mechanical assault and to prevent transepidermal water loss. In infancy, the consequence of this disrupted barrier can be particularly dangerous, at times life-threatening, with increased susceptibility to infection secondary to impaired skin integrity and dramatically increased metabolic demands due to increased epidermal turnover and evaporative heat and water loss.

This article will review the neonatal presentations of selected ichthyoses, describe potential complications and causes of morbidity and mortality, discuss management considerations relevant to the neonatal period, and briefly review diagnosis.

\section{Neonatal Presentations of Selected Ichthyoses Collodion Baby}

Collodion baby, sometimes referred to as 'collodion fetus,' is a common presentation of several congenital ichthyoses, most of which are inherited in an autosomal recessive

\footnotetext{
(c) 2012 Elsevier Inc. All rights reserved.

Address correspondence to Brittany Craiglow, MD, Department of Dermatology, Yale University School of Medicine, PO Box 208059, New Haven, CT 06520, brittany.craiglow@yale.edu.

Conflicts of interest: None

Publisher's Disclaimer: This is a PDF file of an unedited manuscript that has been accepted for publication. As a service to our customers we are providing this early version of the manuscript. The manuscript will undergo copyediting, typesetting, and review of the resulting proof before it is published in its final citable form. Please note that during the production process errors may be discovered which could affect the content, and all legal disclaimers that apply to the journal pertain.
} 
manner, including lamellar ichthyosis (LI), congenital ichthyosiform erythroderma (CIE) and self-healing collodion baby. It is a less severe phenotype when compared with harlequin ichthyosis (described below), yet still has significant associated morbidity and mortality. Collodion babies are often born prematurely and present at birth encased within a shiny, taut, cellophane-like membrane (Figure 1a). The tight skin around the eyes and mouth often leads to ectropion (outturning of the eyelids) and eclabium (eversion of the lips), respectively. After birth, the membrane begins to dry and fissure, eventually leading to complete shedding within the first several weeks of life, at which point the particular clinical manifestations of the given underlying disease begin to develop. Other disorders that can present as a collodion baby include trichothiodystrophy, neutral lipid storage disease, and Sjogren-Larsson syndrome, but these are far less common.

\section{Harlequin Ichthyosis}

Harlequin ichthyosis (HI), also known as 'harlequin baby or 'harlequin fetus,' is an extremely rare form of congenital ichthyosis with a distinct and striking phenotype. $\mathrm{HI}$ is inherited in an autosomal recessive fashion and arises secondary to mutations in the ABCA12 gene ${ }^{1-3}$. Infants with $\mathrm{HI}$ are typically born prematurely and at birth are encased in a markedly thickened, hard stratum corneum, which is often described as armor-like (Figure 1b). Soon after birth this thick casing cracks, resulting in deep red transverse and longitudinal fissures separating thick, yellow, geometric plates of skin. Infants with HI demonstrate ectropion and eclabium, underdeveloped ears and nose, and edematous hands and feet, which are oftentimes enveloped in a mitten-like casing. In the past, HI was almost universally fatal; however, with advanced neonatal intensive care in combination with appropriate skin-specific management, many infants with HI now survive. Infrequently HI may present with a milder phenotype that may appear more similar to a collodion baby or may present with less compact, whitish-yellow thickened scale covering the body, which has been described as 'vernix-like' given its resemblance to vernix caseosa.

\section{X-Linked Ichthyosis}

$\mathrm{X}$-linked ichthyosis (XLI), also known as steroid sulfatase deficiency and recessive X-linked ichthyosis, as the name implies, is inherited in an X-linked recessive fashion. XLI results from either a complete deletion of (majority of cases) or an inactivating mutation in the STS gene, resulting in a deficiency of steroid sulfatase $e^{4}$. Steroid sulfatase is also deficient in the placenta, resulting in low maternal urinary estrogen secretion and low amniotic fluid estrogen, which often leads to insufficient cervical dilation in females and subsequent prolonged or difficult labor, frequently necessitating intervention. Most affected male infants show cutaneous manifestations at birth, with pink or red skin and peeling of large, translucent scales. Over time, the scales become darker with decreased tendency to desquamate. While the skin findings at birth are fairly mild in comparison with other forms of ichthyosis, affected males have an increased risk of cryptorchidism and ocular abnormalities, in particular corneal opacities.

\section{Epidermolytic Ichthyosis}

Epidermolytic ichthyosis (EI), also known as bullous congenital ichthyosiform erythroderma or epidermolytic hyperkeratosis, is an autosomal dominant disorder caused by mutations in the genes KRT1 and KRT10, encoding keratin 1 and keratin 10, respectively 5 . EI presents at birth with blistering and areas of denuded skin (Figure 1c). This presentation may initially be confused with epidermolysis bullosa, staphylococcal scalded skin syndrome or toxic epidermal necrolysis. A similar but milder presentation can be seen in infants with ichthyosis bullosa of Siemens, where blisters tend to be smaller and arise at sites of trauma. Over time, the blistering and denudation seen in EI diminishes, and hyperkeratosis becomes 
the prominent cutaneous manifestation, with dark, thick scales, often in a corrugated or ridged pattern.

\section{Netherton Syndrome}

Netherton syndrome is an autosomal recessive disorder caused by mutations in the SPINK5 gene $^{6}$. Affected individuals present at birth or shortly thereafter with generalized redness, known as erythroderma, and peeling. Other characteristic findings include hair shaft abnormalities, most commonly trichorrhexis invaginata or "bamboo" hair, and elevated $\operatorname{IgE}$ levels. Hair shaft abnormalities can be particularly helpful in distinguishing Netherton syndrome from other disorders with similar clinical presentations, such as congenital ichthyosiform erythroderma.

\section{Complications}

Among neonates with congenital ichthyosis, infants with harlequin ichthyosis, collodion babies, and those with epidermolytic ichthyosis and Netherton syndrome are among those at highest risk for complications during the postnatal period. Beyond complications of prematurity, impaired barrier function serves as the primary source of morbidity and mortality during this time. Increased transepidermal water and heat loss lead to hypernatremic dehydration, other electrolyte imbalances, disrupted thermoregulation and calorie malnutrition. Fissuring and denudation can provide a means of entry for microorganisms, leading to skin infections and sepsis. For neonates with collodion membrane or harlequin ichthyosis, immobilization can result in impaired ventilation, hypoxia and pneumonia as well as difficulty sucking or feeding. In addition, constricting bands of skin can at times compromise perfusion and lead to peripheral edema and ischemia. Finally, infants with ectropion are at risk for exposure keratitis.

\section{Management}

Management of neonates with ichthyosis necessitates a multidisciplinary approach, with family members, nursing staff, neonatologists, dermatologists and, in some cases other specialists, all serving as active participants. In situations where a diagnosis of ichthyosis has been made prenatally, it is critical to prepare family and staff as much as possible education is truly a cornerstone of management. Infants presenting with collodion membrane, HI, erythroderma, or widespread blisters will require admission to the neonatal intensive care unit, whereas infants with milder forms, such as XLI, will likely not require intensive care and in many cases may not even require additional time in the hospital.

Because many infants with ichthyosis are born prematurely, the primary risks or complications are oftentimes those related to prematurity rather than skin; therefore, many of the medical concerns are similar to those that neonatologists manage on a routine basis. Beyond issues that result from prematurity, as noted previously, infants face complications related to impaired barrier function. In this respect, management can be both primary and secondary - that is, directing efforts toward improving barrier function as well as addressing consequences that may arise as a result of its being impaired, such as dehydration, malnutrition, or infection.

The majority of therapies aimed at improving barrier function are fairly simple and straightforward. The mainstays of management include providing a humidified, temperaturecontrolled environment via an isolette, daily bathing with only water or mild cleanser, and frequent, liberal applications of bland emollients such as petrolatum-based products. Listed below are management strategies for a variety of common complications (summarized in Table 1). 


\section{Dehydration, Electrolyte Imbalances, and Increased Metabolic Demands}

As noted above, infants with ichthyosis should be maintained in a humidified incubator. Urinary output, weight, and electrolytes should be monitored very closely and managed with electrolyte repletion or intravenous hydration as necessary. A nutrition consult may be helpful in order to assist with determining caloric requirements. In cases in which obtaining peripheral intravenous access and/or transcutaneous monitoring is difficult due to markedly thickened skin, many recommend placing an umbilical line.

\section{Infection}

Close monitoring for infection is essential. Blood cultures should be drawn in settings of temperature and/or hemodynamic instability, lethargy, irritability or poor feeding.

Superficial wound cultures should be reserved for cases in which purulent discharge is noted or in which there are extensive erosions. Once suspected or identified, all infections must be treated aggressively. The use of prophylactic antibiotics in infants with ichthyosis remains controversial, without any controlled trials present in the literature. Among burn patients, a population with a similarly compromised barrier, the data is mixed, with some data suggesting a beneficial effect ${ }^{7}$, but others indicating no benefit ${ }^{8,9}$ or even a detrimental effect, with prophylactic antibiotics favoring the growth of Pseudomonas aeruginosa ${ }^{10}$. As such, prophylactic antibiotics should be reserved for cases in which there is widespread fissuring or denudation and should only be administered after careful consideration of the potential risks and benefits. In cases of more isolated cutaneous fissures and erosions, topical antibiotics such as mupirocin can be used.

\section{Skin Fragility, Risk of Systemic Absorption of Topical Medications, and Pain Control}

In many cases, neonates with ichthyosis have skin that is fragile and more susceptible to injury from even minor trauma. In order to minimize this risk, careful handling and avoidance of conventional adhesives is recommended. To secure lines and tubing, selfadherent wraps (e.g. Coban ${ }^{\mathrm{TM}}$ ) or gauze should be employed instead of tape whenever possible, especially for infants with blisters or denuded skin. There is also an increased risk of transcutaneous absorption in these infants, particularly in cases of EI or Netherton syndrome, in which there are large areas of denuded skin. Certain topical medications pose serious risk, including silver sulfadiazine cream, which has been linked to agranulocytosis ${ }^{11-13}$ and topical calcineurin inhibitors, e.g. tacrolimus ointment and pimecrolimus cream, which can reach detectable serum levels and lead to toxicity ${ }^{14}$. In addition, topical lactic acid preparations have been associated with lactic acidosis ${ }^{15}$, and topical salicylic acid has been linked to salicylate intoxication ${ }^{16,17}$. Antibacterial soaps or washes such as chlorhexidine have the potential to cause toxicity. For this reason, only bland emollients such as white petrolatum, Vaseline ${ }^{\circledR}$ or Aquaphor ${ }^{\circledR}$ ointment should be used, and infants should be bathed in water only or with very mild soaps or cleansers such as Dove ${ }^{\circledR}$ or Cetaphil ${ }^{\circledR}$. Finally, the skin can serve as a source of pain and discomfort, particularly in cases of extensive fissuring or denudation. As such, it is advisable to provide adequate pain control with acetaminophen, non-steroidal anti-inflammatory medications or narcotics.

\section{Flexibility}

Neonates with HI, and in some cases collodion babies, are at risk for complications related to impaired flexibility, particularly in the early newborn period when the thick encasement has not yet begun to fissure. Such complications may include restrictive lung movement with subsequent risk of pneumonia, difficulty feeding or sucking, contractures, and, in some cases of HI, compartment syndrome. Liberal application of emollients can help to improve pliability, but initially ventilatory support and nasogastric feeding will be universally required for infants with $\mathrm{HI}$ and for some presenting as collodion babies. Orthopedic surgery 
consult may be necessary to assist with monitoring for compartment syndrome, and contractures or constricting bands of skin, such as those often seen on the fingers and toes of infants with HI, may require releasing if perfusion is being compromised, which can be performed by a skilled dermatologist, plastic surgeon or orthopedist. Finally, for infants with HI or, less often, collodion babies, topical or systemic retinoids (e.g., tazarotene or acitretin, respectively) may be considered. Retinoids can be quite beneficial, as they can help to accelerate shedding of the thick scale, but because of the risk for systemic side effects, they should be used with caution and under the supervision of an experienced dermatologist.

\section{Ocular Complications}

Infants must be evaluated for the presence of ectropion, which is commonly seen in cases of collodion baby or HI. If present, ophthalmology consult is recommended. Infants with ectropion are at risk for exposure keratitis, so ensuring that the eyes are adequately lubricated is essential - this can be accomplished with hourly artificial tears or ocular lubricant. Topical antibiotics may be required for treatment of conjunctivitis or corneal abscess.

\section{Family Support}

Parents must be involved in discussions with an interdisciplinary medical team regarding diagnosis, prognosis, and management. In more serious cases, such as infants with HI, advance directives may need to be addressed and ethics committee consultation may need to be considered. Religious counseling should also be made available upon request. For all cases, families should be referred to the patient support network 'FIRST' - Foundation for Ichthyosis and Related Skin Types - for additional education and support. FIRST can also provide clinicians with educational materials or referrals to experts in the field of ichthyosis.

\section{Discharge Planning}

Neonates with ichthyosis will have varying lengths of hospitalization depending on the severity of their cutaneous disease and the degree and extent of complications. Prior to discharge, it is important to ensure that close follow-up is scheduled with consulting services, such as dermatology, nutrition, and genetics, particularly for infants with more severe cases. Parents and caregivers must be educated about skin care and provided with written instructions regarding bathing and emollient use. They should also be reminded about possible systemic absorption or toxicity of certain topical medications and counseled on sun protection, as many of these infants are more susceptible to sunburn. Finally, as mentioned above, we encourage referral to the Foundation for Ichthyosis and Related Skin Types (FIRST), which can serve as a means of continued education and support for patients and families.

\section{Diagnosis}

In some cases, there may be indication of a diagnosis of ichthyosis prior to delivery, such as in cases of XLI, which can be a cause of low unconjugated estriol, levels of which are tested on maternal serum screening during the second trimester. HI may be detected based on ultrasonographic findings. ${ }^{18-20}$ Other methods of prenatal diagnosis of ichthyosis include chorionic villus sampling, fetal skin biopsy, and preimplantation testing ${ }^{21}$.

In the neonatal period, a likely diagnosis can often be made on clinical grounds alone. It is important to note, however, that it is not necessary to make a definitive diagnosis at this point, as management is generally not specific to the particular form of ichthyosis present. Within the first year of life, many cases that were not clear at birth become more clinically recognizable, as patients display the characteristic cutaneous phenotype, such as in EI or 
lamellar ichthyosis, or develop associated findings, for example neurologic findings in the setting of Sjögren-Larsson syndrome.

At times, confirmation of diagnosis can be made in the neonatal period by relatively simple diagnostic tests. Skin biopsy findings are often nonspecific and therefore helpful only in a minority of cases, but the procedure is minimally invasive and can at times provide useful information; EI, for instance, often demonstrates characteristic histologic findings. In cases of suspected Netherton syndrome, a hair mount can be performed to examine for abnormalities, including trichorrhexis invaginata ('bamboo hair,' most common), trichorrhexis nodosa (nodes along the hair shaft), and pili torti (twisted hair), although these are not universally present in infancy. Lipid-containing vacuoles in circulating white blood cells are diagnostic of neutral lipid storage disease, which is also associated with sensorineural deafness, myopathy, and cataracts. In cases where a diagnosis is uncertain or for confirmation of suspected diagnosis, genetic testing can be performed under the direction of a dermatologist and/or geneticist. Testing for mutations known to cause ichthyosis is available in most commercial genetic testing laboratories. Genetic diagnosis becomes particularly useful in the setting of future family planning, and we suspect that it may become more common and important in the coming years, as the genetics of these disorders begins to be better characterized and pathogenesis-based therapies are developed.

\section{Conclusion}

Ichthyosis, while fairly rare, is a condition that requires significant attention in the neonatal period. Successful management of ichthyosis in the newborn can be achieved through a thoughtful, directed, and interdisciplinary approach. An understanding of the disrupted barrier in these patients provides a basis for management, and simple measures, including daily bathing and liberal emollients, serve as mainstays of treatment. The Foundation for Ichthyosis and Related Skin Types (www.firstskinfoundation.org) is an excellent resource for both clinicians and families.

\section{Acknowledgments}

I greatly appreciate Bari Cunningham, MD for significant contributions to this manuscript. I also thank Leonard Milstone, MD for providing photographs and always being willing to lend expertise.

\section{References}

1. Akiyama M, Sugiyama-Nakagiri Y, Sakai K, et al. Mutations in lipid transporter ABCA12 in harlequin ichthyosis and functional recovery by corrective gene transfer. J Clin Invest. 2005; 115:1777-84. [PubMed: 16007253]

2. Kelsell DP, Norgett EE, Unsworth H, et al. Mutations in ABCA12 underlie the severe congenital skin disease harlequin ichthyosis. Am J Hum Genet. 2005; 76:794-803. [PubMed: 15756637]

3. Thomas AC, Cullup T, Norgett EE, et al. ABCA12 is the major harlequin ichthyosis gene. J Invest Dermatol. 2006; 126:2408-13. [PubMed: 16902423]

4. Bonifas JM, Morley BJ, Oakey RE, Kan YW, Epstein EH Jr. Cloning of a cDNA for steroid sulfatase: frequent occurrence of gene deletions in patients with recessive $\mathrm{X}$ chromosome-linked ichthyosis. Proc Natl Acad Sci U S A. 1987; 84:9248-51. [PubMed: 3480541]

5. Rothnagel JA, Dominey AM, Dempsey LD, et al. Mutations in the rod domains of keratins 1 and 10 in epidermolytic hyperkeratosis. Science. 1992; 257:1128-30. [PubMed: 1380725]

6. Chavanas S, Bodemer C, Rochat A, et al. Mutations in SPINK5, encoding a serine protease inhibitor, cause Netherton syndrome. Nat Genet. 2000; 25:141-2. [PubMed: 10835624]

7. Avni T, Levcovich A, Ad-El DD, Leibovici L, Paul M. Prophylactic antibiotics for burns patients: systematic review and meta-analysis. BMJ. 2010; 340:c241. [PubMed: 20156911] 
8. Lee F, Wong P, Hill F, Burgner D, Taylor R. Evidence behind the WHO guidelines: hospital care for children: what is the role of prophylactic antibiotics in the management of burns? J Trop Pediatr. 2009; 55:73-7. [PubMed: 19276147]

9. Ergun O, Celik A, Ergun G, Ozok G. Prophylactic antibiotic use in pediatric burn units. Eur J Pediatr Surg. 2004; 14:422-6. [PubMed: 15630646]

10. Ugburo AO, Atoyebi OA, Oyeneyin JO, Sowemimo GO. An evaluation of the role of systemic antibiotic prophylaxis in the control of burn wound infection at the Lagos University Teaching Hospital. Burns. 2004; 30:43-8. [PubMed: 14693085]

11. Wilson P, George R, Raine P. Topical silver sulphadiazine and profound neutropenia in a burned child. Burns Incl Therm Inj. 1986; 12:295-6. [PubMed: 3719408]

12. Viala J, Simon L, Le Pommelet C, Philippon L, Devictor D, Huault G. Agranulocytosis after application of silver sulfadiazine in a 2-month old infant. Arch Pediatr. 1997; 4:1103-6. [PubMed: 9488745]

13. Jarrett F, Ellerbe S, Demling R. Acute leukopenia during topical burn therapy with silver sulfadiazine. Am J Surg. 1978; 135:818-9. [PubMed: 665909]

14. Allen A, Siegfried E, Silverman R, et al. Significant absorption of topical tacrolimus in 3 patients with Netherton syndrome. Arch Dermatol. 2001; 137:747-50. [PubMed: 11405764]

15. Ramirez ME, Youseef WF, Romero RG, et al. Acute percutaneous lactic acid poisoning in a child. Pediatr Dermatol. 2006; 23:282-5. [PubMed: 16780481]

16. Germann R, Schindera I, Kuch M, Seitz U, Altmeyer S, Schindera F. Life threatening salicylate poisoning caused by percutaneous absorption in severe ichthyosis vulgaris. Hautarzt. 1996; 47:624-7. [PubMed: 8964705]

17. Chiaretti A, Schembri Wismayer D, Tortorolo L, Piastra M, Polidori G. Salicylate intoxication using a skin ointment. Acta Paediatr. 1997; 86:330-1. [PubMed: 9099329]

18. Bongain A, Benoit B, Ejnes L, Lambert JC, Gillet JY. Harlequin fetus: three-dimensional sonographic findings and new diagnostic approach. Ultrasound Obstet Gynecol. 2002; 20:82-5. [PubMed: 12100425]

19. Vohra N, Rochelson B, Smith-Levitin M. Three-dimensional sonographic findings in congenital (harlequin) ichthyosis. J Ultrasound Med. 2003; 22:737-9. [PubMed: 12862276]

20. Holden S, Ahuja S, Ogilvy-Stuart A, Firth HV, Lees C. Prenatal diagnosis of Harlequin ichthyosis presenting as distal arthrogryposis using three-dimensional ultrasound. Prenat Diagn. 2007; 27:566-7. [PubMed: 17385787]

21. Fassihi H, Eady RA, Mellerio JE, et al. Prenatal diagnosis for severe inherited skin disorders: 25 years' experience. Br J Dermatol. 2006; 154:106-13. [PubMed: 16403102] 

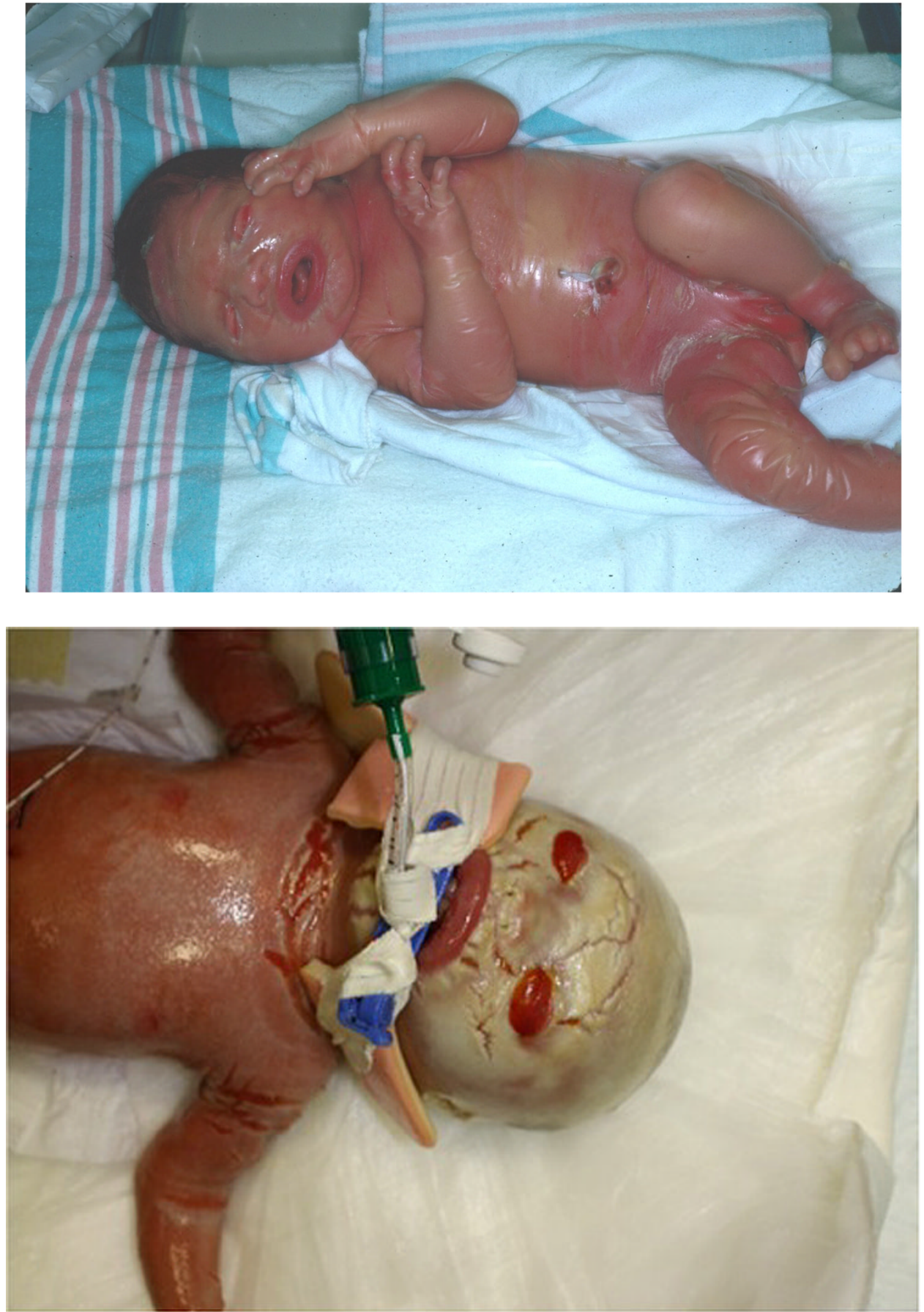


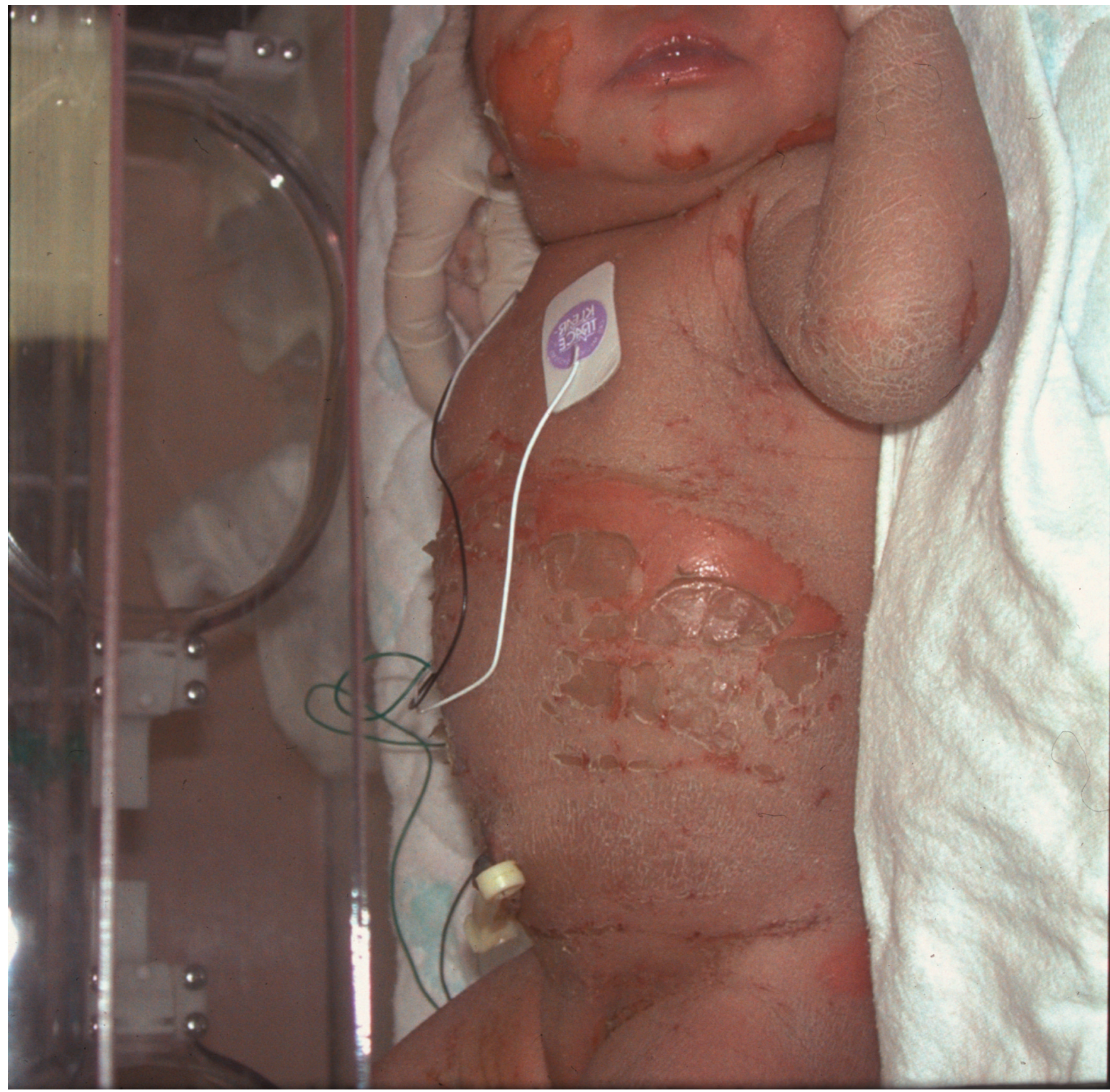

Figure 1.

(a) Collodion baby. Infant with taut, shiny, cellophane-like membrane, ectropion and eclabium. Courtesy of Leonard Milstone, MD. (b) Harlequin ichthyosis. Thick stratum corneum with fissures, marked ectropion, and eclabium. Courtesy of Yale Dermatology Residents' slide collection. (c) Epidermolytic ichthyosis. Superficial erosions on a background of hyperkeratosis. Courtesy of Leonard Milstone, MD 


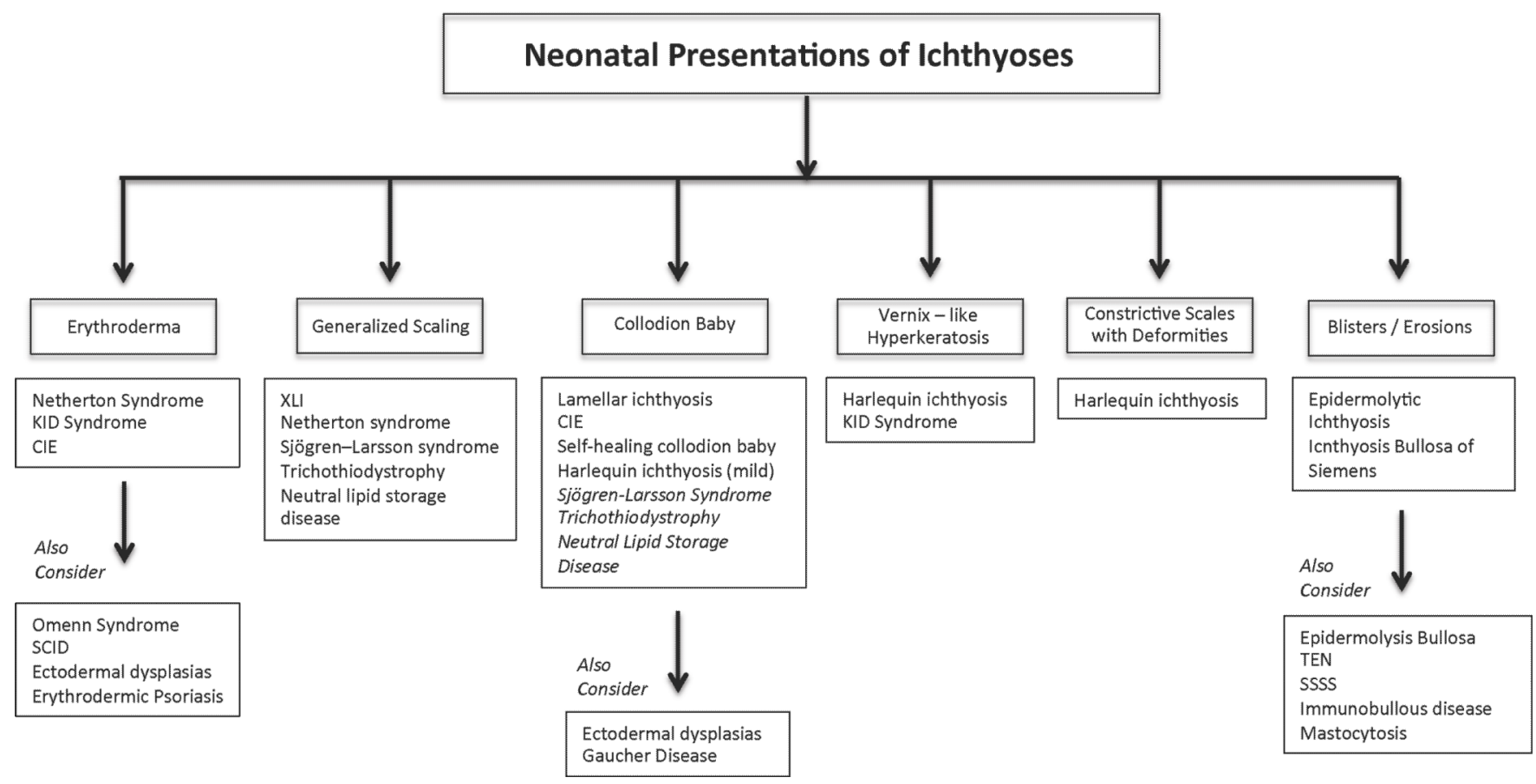

FIGURE 2.

Neonatal presentations of ichthyoses.

Figure presents the spectrum of possible presentations of ichthyosis in the neonate and the possible underlying forms of ichthyosis. In some cases, disorders beyond ichthyosis must be considered ("also consider"). Sjögren-Larsson syndrome, trichothiodystrophy, and neutral lipid storage disease are italicized, as they rarely present as collodion babies.

Abbreviations: KID syndrome $=$ keratitis-ichthyosis-deafness syndrome; $\mathrm{CIE}=$ congenital ichthyosiform erythroderma; SCID = severe combined immunodeficiency; TEN = toxic epidermal necrolysis, SSSS = staphylococcal scalded skin syndrome 


\section{TABLE 1}

Normal skin function, possible complications in ichthyosis, and related management strategies.

Column 1 lists functions of normal skin; column 2 lists related potential complications in ichthyosis, and Column 3 provides related management strategies.

Adapted with permission from Cunningham, B. "Management of Ichthyosis in the

Neonate"www.firstskinfoundation.org.

\begin{tabular}{|c|c|c|}
\hline NORMAL SKIN & ICHTHYOSIS & MANAGEMENT STRATEGIES \\
\hline $\begin{array}{l}\text { Important role in innate } \\
\text { immunity; barrier against } \\
\text { infection }\end{array}$ & $\begin{array}{l}\text { High risk for bacterial } \\
\text { infection and sepsis }\end{array}$ & $\begin{array}{l}\text { Close monitoring of vital signs } \\
\text { Blood cultures if temperature/hemodynamic instability, lethargy } \\
\text { Aggressive treatment of infections } \\
\text { Topical antibiotics to fissures } \\
\text { Reverse isolation }\end{array}$ \\
\hline $\begin{array}{l}\text { Maintenance of fluid- } \\
\text { electrolyte balance; barrier } \\
\text { against excessive losses }\end{array}$ & $\begin{array}{l}\text { Increased water loss; risk of } \\
\text { dehydration and electrolyte } \\
\text { imbalance }\end{array}$ & $\begin{array}{l}\text { Monitor fluid balance by intake, urinary output, daily weights } \\
\text { Regular serum electrolytes with repletion } \\
\text { Humidified incubator } \\
\text { Nutrition Consult } \\
\text { Intravenous hydration }\end{array}$ \\
\hline $\begin{array}{l}\text { Barrier against chemical and } \\
\text { mechanical assaults }\end{array}$ & $\begin{array}{l}\text { Risk of systemic absorption } \\
\text { of topicals; skin fragility }\end{array}$ & $\begin{array}{l}\text { Bland emollients only } \\
\text { Avoid silver sulfadiazine, lactic acid and salicylic acid preparations, } \\
\text { antibacterial soaps } \\
\text { Minimal handling } \\
\text { Avoid conventional adhesives; use self-adherent wraps or gauze }\end{array}$ \\
\hline $\begin{array}{l}\text { Flexible and pliable to allow } \\
\text { full range of motion of body } \\
\text { parts }\end{array}$ & $\begin{array}{l}\text { Risk of restriction of lung } \\
\text { movement; difficulty } \\
\text { sucking/feeding; } \\
\text { contractures; compartment } \\
\text { syndrome }\end{array}$ & $\begin{array}{l}\text { Respiratory support } \\
\text { Nasogastric feeding } \\
\text { Liberal emollients, daily bathing and/or retinoids to thin scale } \\
\text { Monitor perfusion and compartment pressure } \\
\text { Orthopedic or plastic surgery consult in cases of contractures or } \\
\text { constriction } \\
\text { Ophthalmology consult in cases of ectropion }\end{array}$ \\
\hline
\end{tabular}

